\title{
XMM-Newton observations of five INTEGRAL sources located towards the Scutum Arm
}

\author{
A. Bodaghee and J. A. Tomsick \\ Space Sciences Laboratory, 7 Gauss Way, University of California, Berkeley, CA 94720, USA \\ bodagheeessl.berkeley.edu \\ and \\ J. Rodriguez \\ Laboratoire AIM, CEA/IRFU - Université Paris Diderot - CNRS/INSU, \\ CEA DSM/IRFU/SAp, Centre de Saclay, F-91191 Gif-sur-Yvette, France
}

\begin{abstract}
Results are presented for XMM-Newton observations of five hard X-ray sources discovered by $I N$ TEGRAL in the direction of the Scutum Arm. Each source received $\gtrsim 20 \mathrm{ks}$ of effective exposure time. We provide refined X-ray positions for all 5 targets enabling us to pinpoint the most likely counterpart in optical/infrared archives. Spectral and timing information (much of which are provided for the first time) allow us to give a firm classification for IGR J18462-0223 and to offer tentative classifications for the others. For IGR J18462-0223, we discovered a coherent pulsation period of $997 \pm 1 \mathrm{~s}$ which we attribute to the spin of a neutron star in a highly-obscured $\left(N_{\mathrm{H}}=2 \times 10^{23} \mathrm{~cm}^{-2}\right)$ high-mass X-ray binary (HMXB). This makes IGR J18462-0223 the seventh supergiant fast X-ray transient (SFXT) candidate with a confirmed pulsation period. IGR J18457+0244 is a highly-absorbed $\left(N_{\mathrm{H}}=8 \times 10^{23} \mathrm{~cm}^{-2}\right)$ source in which the possible detection of an iron line suggests an active galactic nucleus (AGN) of type Sey-2 situated at $z=0.07(1)$. A periodic signal at $4.4 \mathrm{ks}$ could be a quasi-periodic oscillation which would make IGR J18457+0244 one of a handful of AGN in which such features have been claimed, but a slowlyrotating neutron star in an HMXB can not be ruled out. IGR J18482+0049 represents a new obscured HMXB candidate with $N_{\mathrm{H}}=4 \times 10^{23} \mathrm{~cm}^{-2}$. We tentatively propose that IGR J18532+0416 is either an AGN or a pulsar in an HMXB system. The X-ray spectral properties of IGR J18538-0102 are consistent with the AGN classification that has been proposed for this source.
\end{abstract}

Subject headings: accretion, accretion disks ; gamma-rays: general ; stars: neutron ; X-rays: binaries ; X-rays: individual (IGR J18457+0244, IGR J18462-0223, IGR J18482+0049, IGR J18532+0416, IGR J18538-0102)

\section{Introduction}

Surveys by INTEGRAL have enabled the discovery of hundreds of new high-energy sources (e.g., Bird et al. 2010; Krivonos et al. 2010). While INTE$G R A L$ has proven adept at finding new sources, the position error radii are on the order of a few arcminutes. These are clearly too large to permit the identification of a single counterpart in the optical and infrared bands. Establishing the nature of the optical/IR counterpart is a crucial element in helping to catego- rize an INTEGRAL Gamma-Ray source (IGR)1 into one of the many groups of high-energy emitters.

Hence, the classification of IGRs depends on subsequent observations with $\mathrm{X}$-ray focusing telescopes which provide position accuracies of a few arcseconds (e.g., Rodriguez et al. 2010). What these follow-up observations have shown is that some IGRs belong to a previously-rare subclass of high-mass X-ray binary (HMXB) called supergiant X-ray binaries (SGXBs: e.g., Walter et al. 2006, and references therein). Of

\footnotetext{
${ }^{1}$ a comprehensive list of IGRs and their properties can be found at http://irfu.cea.fr/Sap/IGR-Sources
} 


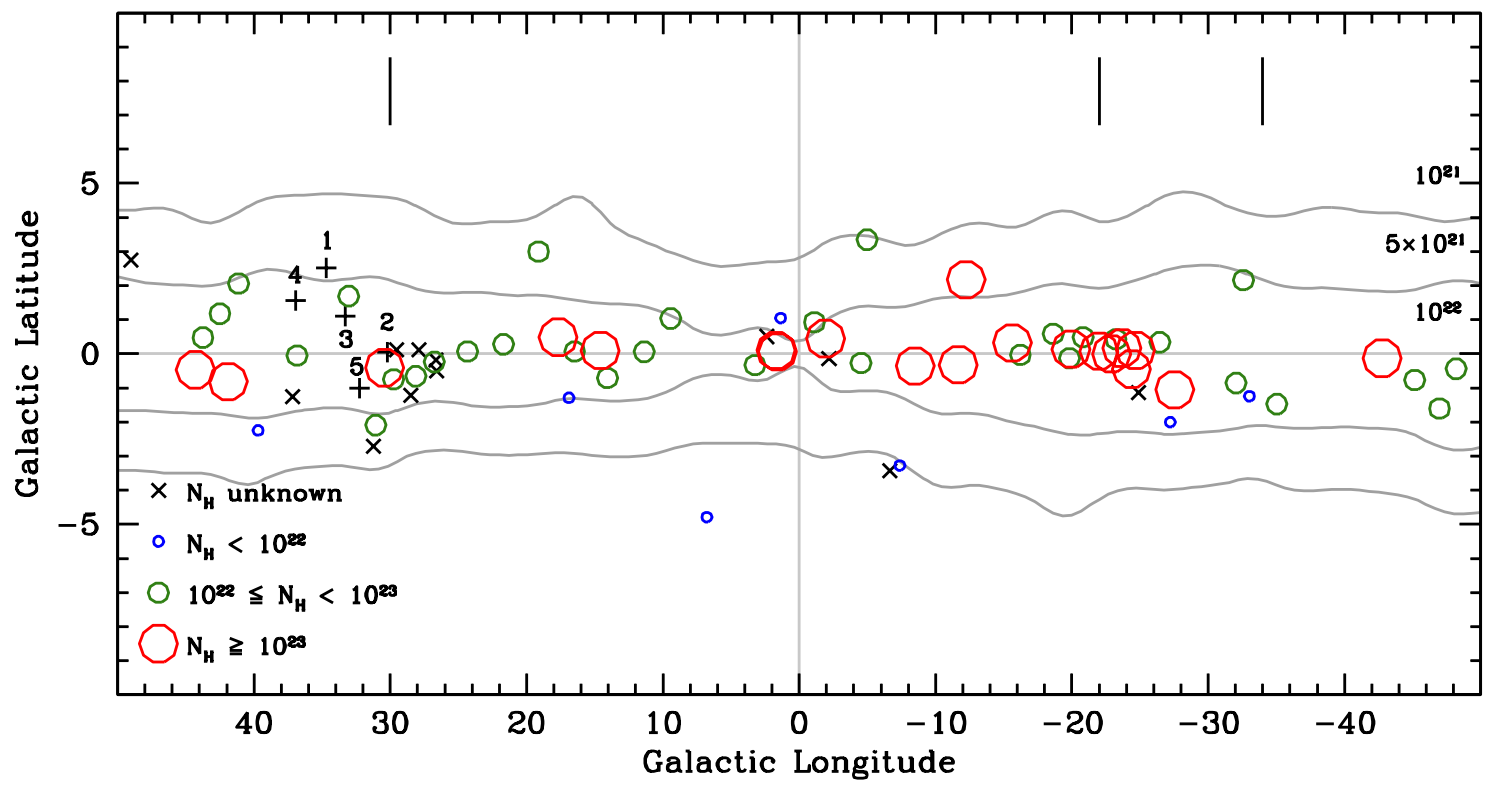

Fig. 1.- The spatial distribution of HMXBs in Galactic coordinates with symbol size proportional to the X-ray measured column density as reported in the literature. The contours represent cumulative line-of-sight absorption levels of $10^{21}, 5 \times 10^{21}$, and $10^{22} \mathrm{~cm}^{-2}$ (Dickey \& Lockman 1990). The vertical bars denote the tangents to the (left to right) Scutum, Inner Perseus, and Norma arms from Vallée (2008). The five targets in this study (which are unclassified or tentatively classified) are labeled with crosses and are numbered 1 through 5 in order of increasing R.A. (see Table 1). The $N_{\mathrm{H}}$ values are known for some of the targets (see Section3).

the 50 IGR HMXBs, 26 of them are SGXBs representing new additions to a subclass that once contained less than a dozen members (Liu et al. 2000): the most prominent examples of which are Vela X-1 and GX 301-2. A common characteristic of SGXBs is their high intrinsic column $\left(N_{\mathrm{H}} \geq 5 \times 10^{22} \mathrm{~cm}^{-2}\right)$ which suggests that the compact accretor is embedded in the dense winds shed by its supergiant companion star. The most extreme case is IGR J16318-4848 with the largest $N_{\mathrm{H}}$ of any X-ray source known in the Galaxy $\left(N_{\mathrm{H}} \sim 2 \times 10^{24} \mathrm{~cm}^{-2}\right.$, Matt \& Guainazzi 2003). The typical $N_{\mathrm{H}}$ value of these systems is a few $\times 10^{23} \mathrm{~cm}^{-2}$.

The spatial distribution of obscured HMXBs in the inner Milky Way appears to be asymmetric about the Galactic Center (GC) Bodaghee et al. 2007). In the inner quadrant of the Galactic Plane (Fig.11), there are 37 HMXBs (with and without $N_{\mathrm{H}}$ measurements) at positive longitudes, and an equivalent number (34) at negative longitudes. Among HMXBs whose $N_{\mathrm{H}}$ values are known to be less than $10^{23} \mathrm{~cm}^{-2}$, the left-right distribution is also symmetrical about the GC: 21 vs.
18. Thus, the asymmetry is only evident for the most obscured systems $\left(N_{\mathrm{H}} \geq 10^{23} \mathrm{~cm}^{-2}\right)$ : there are around half as many obscured HMXBs (7) at positive longitudes as there are at negative longitudes (13). On the other hand, we have an incomplete picture of the obscured HMXB population in the direction of the Scutum Arm. There are unclassified IGRs (including HMXB candidates) in this region. If some of these are shown to be obscured HMXBs, then it will level the Galactic distribution, which might indicate that the asymmetry was the result of an observational bias rather than being due to, e.g., a possible evolutionary difference between the hard X-ray populations of the arms.

In order to help complete, at least partially, the sample of obscured HMXBs, we obtained XMM-Newton observations of five unclassified (or tentatively classified) IGRs that are located in the direction of the Scutum Arm. Their persistent emission above $20 \mathrm{keV}$, lack of known X-ray counterpart below $10 \mathrm{keV}$, and location close to the Galactic Plane make them good can- 
Table 1: Journal of XMM-Newton observations. The effective exposure time represents the time dedicated to the source position (with EPIC-pn) after the filtering and cleaning processes.

\begin{tabular}{lccccc}
\hline \hline Target & Spacecraft Rev. & Obs. ID & Start Time (UTC) & End Time (UTC) & Eff. Exp. (ks) \\
\hline IGR J18457+0244 & 2075 & 0651680201 & $2011-04-09 T 05: 18: 17$ & $2011-04-09 T 12: 41: 58$ & 25.036 \\
IGR J18462-0223 & 2080 & 0651680301 & $2011-04-18 T 08: 52: 18$ & $2011-04-18 T 17: 44: 32$ & 31.937 \\
IGR J18482+0049 & 2079 & 0651680401 & $2011-04-16 T 18: 39: 11$ & $2011-04-17 T 01: 06: 24$ & 23.236 \\
IGR J18532+0416 & 2077 & 0651680501 & $2011-04-12 T 12: 10: 49$ & $2011-04-12 T 17: 56: 41$ & 20.752 \\
IGR J18538-0102 & 2081 & 0651680101 & $2011-04-20 T 14: 44: 35$ & $2011-04-20 T 21: 48: 30$ & 25.437 \\
\hline
\end{tabular}

didates for being obscured HMXBs. We present our observations and analysis methods in Section 2 Results for individual sources are discussed and summarized in Sections 34

\section{Observations \& Analysis}

Our target list consists of five hard X-ray sources located towards the Scutum Arm. These sources were observed by the XMM-Newton (Jansen et al. 2001; Strüder et al. 2001; Turner et al. 2001) telescope during April, 2011, for approximately $30 \mathrm{ks}$ each (Observation IDs: 0651680101-0651680501; PI: Bodaghee). Table 1 provides the observation logs. All data reduction and analysis steps were performed using HEASOFT 6.11 and the Science Analysis System (SAS) 11.0.0.

We reprocessed the MOS and pn events files using emproc and epproc, respectively. To identify epochs with a high particle background, we used evselect to create single-event (i.e., PATTERN==0) light curves above $10 \mathrm{keV}$ for MOS $1 / 2$ and between 10 and $12 \mathrm{keV}$ for pn which covered the full field of view (FOV) of each instrument. For the spectral analysis, we excluded epochs in which the particle background was higher than 1 count per second (cps). The good time intervals (GTIs) that remained were then used in evselect to produce filtered event files for each instrument. Time stamps from the satellite reference frame were converted to that of the Solar System's barycenter with the barycen tool.

From these cleaned event lists, we generated images for MOS $1 / 2$ and for pn over the full FOV and covering the full spectrum of each instrument. The source extraction region consisted of a circle with a radius of $25^{\prime \prime}$ ( $=500$ pixels in the unbinned image) centered on the brightest pixel. This radius was chosen to be wide enough to encompass most of the source photons (this corresponds to an encircled energy fraction of $\sim 80 \%$ for the PSF from MOS and pn according to the SAS user's manual2). However, since some of our targets are located near the edge of a CCD, we also wanted this radius to be restrictive enough so as to avoid collecting photons from an adjacent CCD. For the background extraction region, we used a circle of radius equal to $70^{\prime \prime}$ (1400 pixels) from a source-free region located on the same CCD as the target source. Whenever possible, this background region was chosen to be more than $1^{\prime}$ away from the target source, at an equivalent off-axis angle as the source region in MOS, or centered at the same RAW_Y pixel in pn (i.e., the center of the background region is shifted horizontally in the detector space with respect to the center of the source region).

Figure 2 presents the MOS1 images of the fields centered on the INTEGRAL-ISGRI positions of the five targets from Bird et al. (2010). We ran edetect on the cleaned event files of each instrument and produced lists of detected sources in several energy bands. The output list from edetect includes a dozen or more source detections, a few of which are located inside the $90 \%$-confidence ISGRI error circle. Spectra were extracted for all sources detected inside the most accurate ISGRI position available: the radius of the error circle is $1: 6$ for IGR J18462-0223 (Grebenev \& Sunyaev 2010), and $\sim 4^{\prime}$ for the others (Bird et al. 2010). Within each ISGRI circle, we identified a single XMM-Newton counterpart (which happened to be the brightest one) whose spectrum was consistent with the source being an IGR, i.e., a hard power law $(\Gamma \sim 0-2)$ with some photoelectric absorption $\left(N_{\mathrm{H}} \geq 10^{21} \mathrm{~cm}^{-2}\right)$. We were also able to compare the spectral parameters (and coordinates) with those from previous soft X-ray observations (Section 3).

Once the correct X-ray counterpart was identified

\footnotetext{
${ }^{2}$ http://xmm.esac.esa.int/external/xmm_user_support/ documentation/uhb_2.5/node17.html
} 


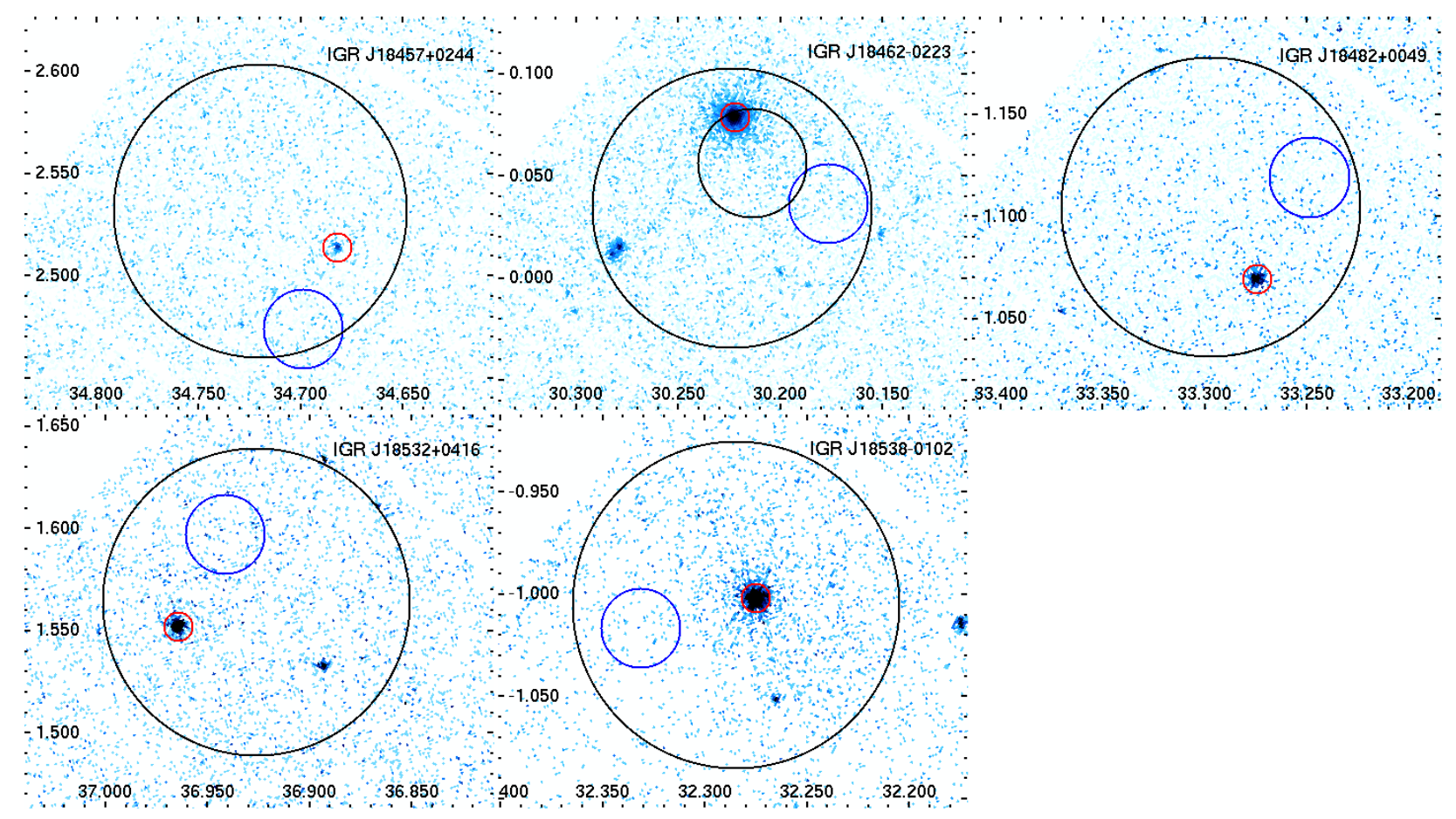

Fig. 2.- EPIC-MOS1 images $(0.5-10 \mathrm{keV})$ in Galactic coordinates of the five targets in this study. The black circles represent the INTEGRAL-ISGRI 90\%-confidence circles: the large circles are from Bird et al. (2010), while for IGR J18462-0223, the more accurate ISGRI position from Grebenev \& Sunyaev (2010) is also shown. The source and background extraction regions are presented as red and blue circles, respectively. North is up and East is left.

Table 2: X-ray positions (J2000) from XMM-Newton for the IGR sources in this program. The positions (90\% confidence radius of 2".5) represent an average of the positions found by running edetect on pn, MOS1, and MOS2. The nearest infrared counterpart from 2MASS (Skrutskie et al. 2006) is listed, as is its offset with the X-ray position (in arcseconds).

\begin{tabular}{|c|c|c|c|c|c|c|}
\hline Source Name & R.A. & Dec. & $\bar{l}$ & $\bar{b}$ & counterpart candidate & offset (") \\
\hline IGR J18457+0244 & $18^{\mathrm{h}} 45^{\mathrm{m}} 40 \mathrm{~s} 30$ & $+02^{\circ} 42^{\prime} 11^{\prime \prime} \cdot 2$ & 34.682 & +2.514 & 2MASS J18454039+0242088 & 2.7 \\
\hline IGR J18462-0223 & $18^{\mathrm{h}} 46^{\mathrm{m}} 12 \mathrm{~s} 68$ & $-02^{\circ} 22^{\prime} 29^{\prime \prime} \cdot 3$ & 30.223 & +0.079 & 2MASS J18461279-0222261 & 3.4 \\
\hline IGR J18482+0049 & $18^{\mathrm{h}} 48^{\mathrm{m}} 15^{\mathrm{s}} 32$ & $+00^{\circ} 47^{\prime} 34^{\prime \prime} \cdot 9$ & 33.275 & +1.070 & $8481540+0047332$ & 2.0 \\
\hline IGR J18532+0416 & $18^{\mathrm{h}} 53^{\mathrm{m}} 15^{\mathrm{s}} 83$ & $+04^{\circ} 17^{\prime} 48^{\prime \prime} \cdot 5$ & 36.965 & +1.553 & 17481 & 2.9 \\
\hline IGR J18538-0102 & $18^{\mathrm{h}} 53^{\mathrm{m}} 48 \mathrm{~s} .42$ & $-01^{\circ} 02^{\prime} 28^{\prime \prime} \cdot 3$ & 32.275 & -1.002 & 2MASS J18534847-0102295 & 1.6 \\
\hline
\end{tabular}

for each IGR source, its coordinates from MOS1/2 and pn were averaged and this position is reported in Table 2 (equinox 2000.0 is used throughout this work). According to the latest calibration documents (XMMSOC-CAL-TN-00183), the position uncertainty for EPIC is $1 " .5$ (at $68 \%$ confidence) which is dominated by systematics. Thus, we have adopted a value of $2 \prime .5$ (at 90\% confidence) for the source position uncer-

\footnotetext{
${ }^{3}$ http://xmm2.esac.esa.int/external/xmm_sw_cal/calib
}

tainty.

Figure 3 presents the light curve for the particle background, as well as for the source and background extraction regions as defined above. Light curves and spectra were created for the source and background regions in $0.1-10 \mathrm{keV}(\operatorname{PATTERN} \leq 12)$ for $\mathrm{MOS} 1 / 2$ ( $5 \mathrm{~s}$ resolution), and in $0.13-15 \mathrm{keV}($ PATTERN $\leq 4)$ for pn ( $0.1 \mathrm{~s}$ resolution). We accounted for the difference in the size of the extraction areas by run- 

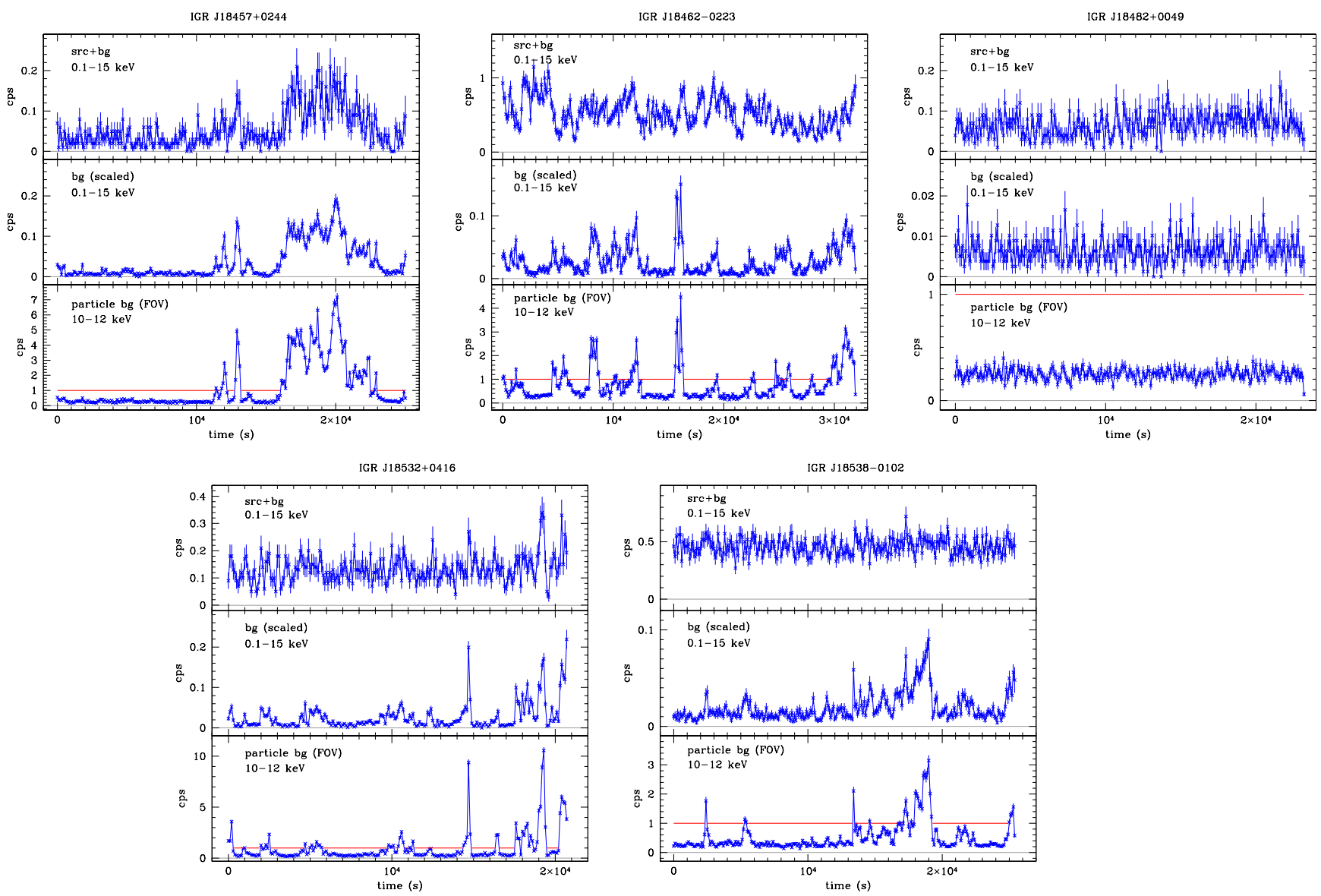

Fig. 3.- EPIC-pn light curves with a time time resolution of $100 \mathrm{~s}$. In each figure, the top and middle panels present the raw count rates in the $0.13-15-\mathrm{keV}$ range from the source (not corrected for the background) and background extraction regions (scaled to the size of the source region). The bottom panel shows the count rate between 10 and $12 \mathrm{keV}$ in the full field of view (FOV) attributed to the particle background. Only time intervals during which the particle background rate was below $1 \mathrm{cps}$ (represented by the red line) are considered in the spectral analysis.

ning epiclccorr on the light curves (which subtracted this scaled background count rate from the source count rate while correcting for exposure, PSF, and vignetting effects), and by running backscale on the spectra. Spectral RMF and ARF files were generated using rmfgen and arfgen, respectively. These files were grouped with the source and background spectral files by employing specgroup with a minimum of 20 counts per bin and a maximum oversampling factor of 3. Figures 4 and 5 present the light curves and spectra, respectively, of the five sources in our study. We searched for periodic signals in the raw (i.e., not corrected for the background) source light curves from pn using both efsearch and the fast algorithm for Lomb-Scargle periodograms developed by Press \& Rybicki (1989) with error analysis from Horne \& Baliunas (1986). Spectral fits employed the abundances of Wilms et al. (2000) and the photo-ionization cross sections of Balucinska-Church \& McCammon (1992). In the following section, we will discuss the results of each source in detail.

\section{Results \& Discussion}

\subsection{IGR J18457+0244}

Soon after the discovery of this source by Bird et al. (2010), it was followed up by a Swift observation in 

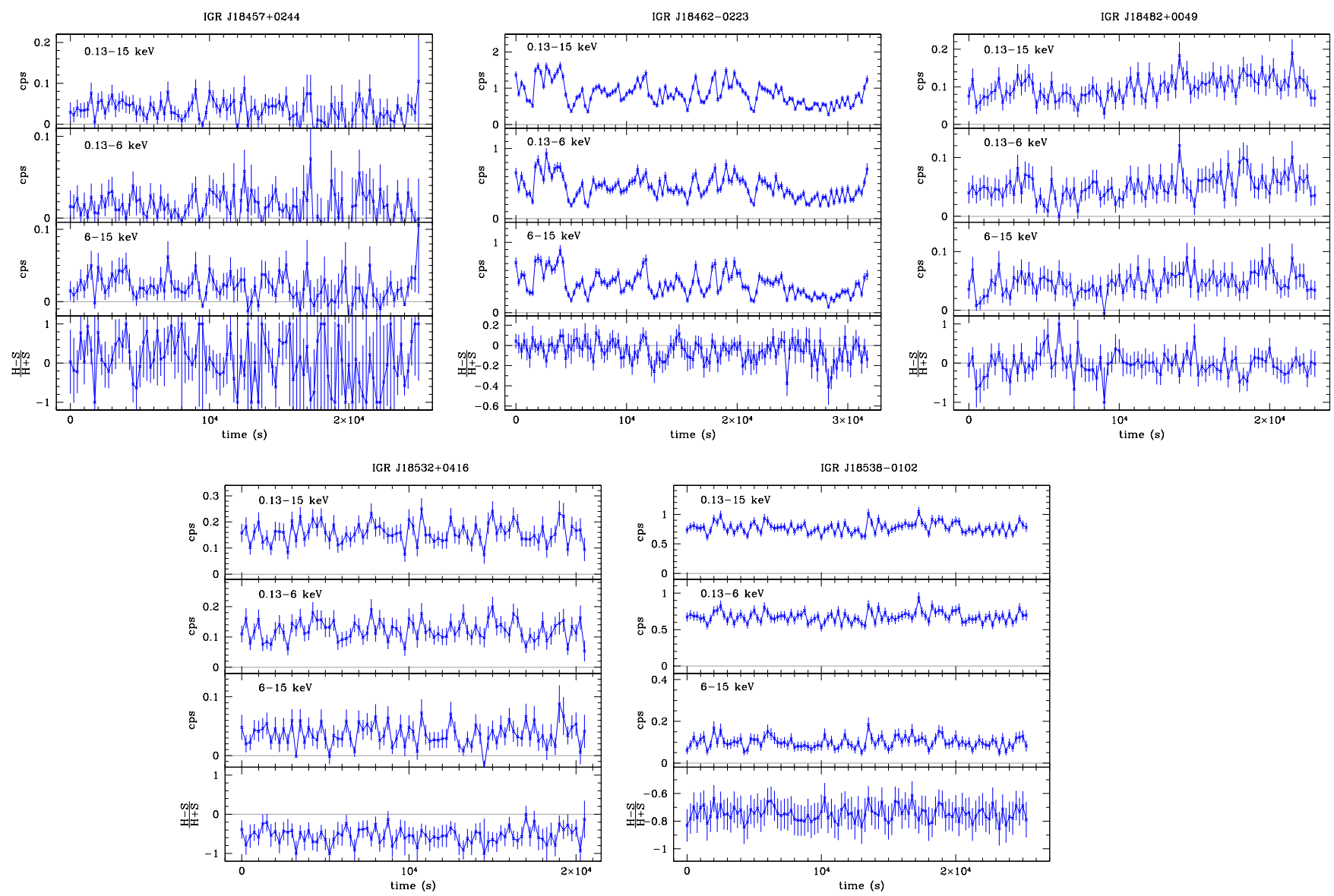

Fig. 4.- Background-subtracted and exposure-corrected light curves of the five target sources taken with EPIC-pn in the full energy range (0.13-15 keV), and in the soft (S: $0.13-6 \mathrm{keV})$ and hard bands (H: 6-15 keV). The time resolution is $250 \mathrm{~s}$. The hardness ratio is defined as $\frac{H-S}{H+S}$.

which a soft X-ray counterpart was detected at the 4- $\sigma$ level in the $0.3-10-\mathrm{keV}$ band (Landi et al. 2010). The $\mathrm{X}$-ray position that we obtain for IGR J18457+0244 is R.A. $=18^{\mathrm{h}} 45^{\mathrm{m}} 40 \mathrm{~s} 30$ and Dec. $=+02^{\circ} 42^{\prime} 11^{\prime \prime} \cdot 2$. Our XMM-Newton position is $4.9^{\prime \prime}$ away from the Swift position listed in Landi et al. (2010), but the latter has a reported uncertainty radius of $6^{\prime \prime}$ so the positions are statistically compatible. The nearest infrared source in the 2MASS catalog (Fig.6), 2MASS J18454039+0242088, is $2.8^{\prime \prime}$ away from the XMM-Newton position (i.e., just outside the $90 \%$ confidence radius of 2 '.5), and it has $J, H$, and $K$ band magnitudes of $\geq 16.2,15.3 \pm 0.1$, and $14.6 \pm 0.1$, respectively (Skrutskie et al. 2006). There are no catalogued objects from other wavelengths inside the XMM-Newton error circle.
Figure4 4 presents the pn light curve for IGR J18457+0244. Searching for periodicities in this light curve uncovers a potential signal at a period of $4400 \mathrm{~s}$ ( 8 bins per trial period) with $\chi^{2}=56$ (6- $\sigma$ significance, not corrected for the number of trials) and a pulse fraction $\left(\equiv \frac{I_{\max }-I_{\min }}{I_{\max }+I_{\min }}\right)$ of $32 \% \pm 7 \%$. Large wings surround the main peak due to the long period of this potential signal which means that only 5 cycles are contained within the full $25-\mathrm{ks}$ observation. The $\chi^{2}$ distribution and folded light curve are presented in Fig.7 This signal is only seen with efsearch, and not with the Lomb-Scargle periodogram nor in the power spectrum. Using the Leahy-normalized power spectrum (Leahy et al. 1983), we determined a 90\% confidence upper limit of 25.33 on the Leahy Power (van der Klis 1989) which converts to an upper limit of $14 \%$ on the 

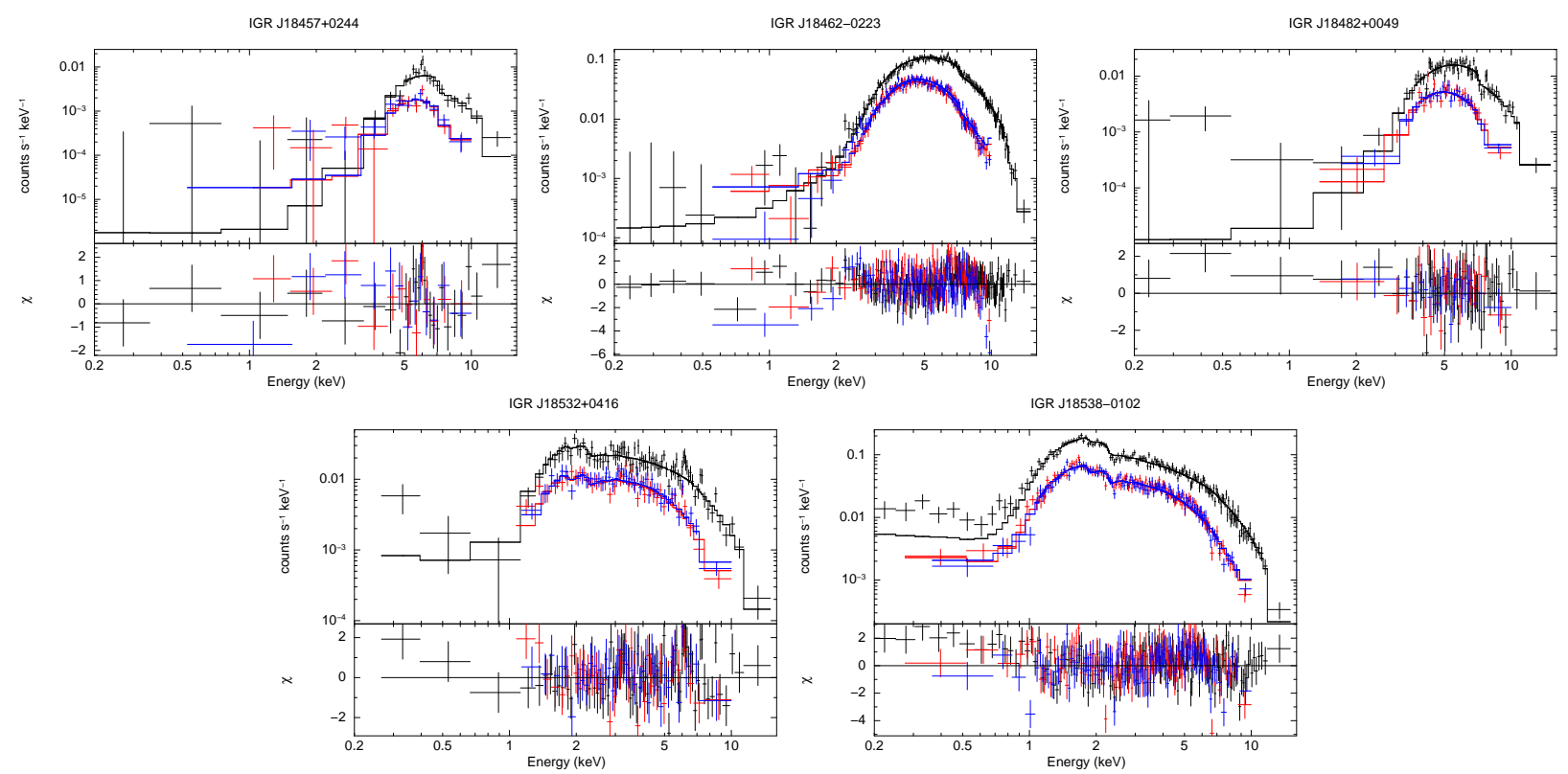

Fig. 5. - Spectra (corrected for the background) in the $0.13-15-\mathrm{keV}$ band of the five sources as gathered with pn (black), MOS1 (red), and MOS2 (blue). Each bin collects a minimum of 20 counts, and the spectra have been modeled with an absorbed power law whose parameters are given in Table3.

fractional r.m.s. expected for a periodic signal due to noise (i.e., less than the pulse fraction that we measured).

We note that aperiodic variability on a $\sim 4-\mathrm{ks}$ timescale (i.e., a large fraction of the observation duration) would also lead to multiple wings surrounding a central peak in the $\chi^{2}$ distribution, so this can not be excluded. Thus, an intriguing possibility is that the weak periodicity at $4.4 \mathrm{ks}$ is a quasi-periodic oscillation (QPO) related to the motion of material along the innermost stable orbit of an accretion disk. Such QPOs are a common feature of the power spectrum of black hole binaries. They have been confirmed in one AGN thus far (RE J1034+396) where the peak frequency of the QPO is $2.7 \times 10^{-4} \mathrm{~Hz}$ (i.e., 3.7-ks periodicity) (Gierliński et al. 2008), and they have been proposed in other AGN as well (e.g., Espaillat et al.,2008; Gupta et al. 2009; Lachowicz et al. 2009). These frequencies are similar in scale to the 4.4-ks periodicity that we see in IGR J18457+0244.

A power law fit to the $\mathrm{X}$-ray spectrum (Fig. 5) yields a column density $\left(N_{\mathrm{H}}=\left(84_{-16}^{+20}\right) \times 10^{22} \mathrm{~cm}^{-2}\right)$ that is larger than that expected along the line of sight Kalberla et al. 2005), which suggests that it is intrinsic to the system, and a steep photon index $(\Gamma=$ $2.3 \pm 0.7)$. These parameters are in good agreement with those from Swift-XRT (Landi et al. 2010), despite the fact that our observed (i.e., not corrected for absorption) $0.5-10-\mathrm{keV}$ flux is $4.6 \times 10^{-13} \mathrm{erg} \mathrm{cm}^{-2} \mathrm{~s}^{-1}$ $\left(=5.5 \times 10^{33}\left[\frac{d}{10 \mathrm{kpc}}\right]^{2} \mathrm{erg} \mathrm{s}^{-1}\right)$, i.e., lower than that of the $2-10-\mathrm{keV}$ flux observed with XRT. The quality of the fit is good $\left(\chi_{v}^{2} / \mathrm{dof}=1.13 / 49\right)$, but a few residuals around $6 \mathrm{keV}$ hint at an iron fluorescence line. Adding a Gaussian to the model reduces the $\chi_{v}^{2} /$ dof to $0.85 / 46$. The line energy is $6.01_{-0.04}^{+0.06} \mathrm{keV}$ with an equivalent width $(E W)$ of $300 \pm 150 \mathrm{eV}$. A good fit $\left(\chi_{v}^{2} / \mathrm{dof}=0.92 / 46\right)$ is also obtained with an absorbed blackbody model that includes a Gaussian profile for the possible iron line. In this case, the blackbody temperature is $2.1_{-0.4}^{+0.6} \mathrm{keV}$, and $N_{\mathrm{H}}=\left(54_{-14}^{+15}\right) \times 10^{22} \mathrm{~cm}^{-2}$. Modeling the spectrum with a cutoff power law or a thermal Comptonization model (comptt in Xspec) returned upper limits on the spectral parameters. These results are summarized in Table 3 .

Assuming that the rest energy of the iron $K \alpha$ line is $6.41 \mathrm{keV}$, this would place IGR J18457+0244 at a redshift of $z=0.07(1)$, suggesting an active galactic nucleus (AGN) most likely of the Seyfert- 2 class given its large absorbing column. The corresponding (unabsorbed) $\mathrm{X}$-ray luminosity in the $0.5-10-\mathrm{keV}$ band is $\left(9_{-2}^{+3}\right) \times 10^{43} \mathrm{erg} \mathrm{s}^{-1}$, assuming $H_{\circ}=70 \mathrm{~km} \mathrm{~s}^{-1} \mathrm{Mpc}^{-1}$ and a flat cosmology. We point out that other than the 
TABLE 3

SPECTRAL PARAMETERS FROM MODELS FIT TO THE PN AND MOS SPECTRA OF THE FIVE TARGETS.

\begin{tabular}{|c|c|c|c|c|c|c|c|c|c|c|c|}
\hline Source Name & Xspec Model & C & $\begin{array}{c}N_{\mathrm{H}} \\
10^{22} \mathrm{~cm}^{-2}\end{array}$ & $\Gamma$ or $\tau$ & $\begin{array}{c}E_{\text {cut }} \text { or } T_{0} \\
\mathrm{keV}\end{array}$ & $\begin{array}{l}k T \\
\mathrm{keV}\end{array}$ & $\begin{array}{c}E(\mathrm{~K} \alpha) \\
\mathrm{keV}\end{array}$ & $\begin{array}{c}E W(\mathrm{~K} \alpha) \\
\mathrm{eV}\end{array}$ & $\begin{array}{c}\text { Norm. }(\mathrm{K} \alpha) \\
10^{-6} \text { photons cm } \mathrm{cm}^{-2} \mathrm{~s}^{-1}\end{array}$ & $\begin{array}{c}{ }_{\text {unabs }} F_{0.5-10 \mathrm{keV}} \\
10^{-12} \mathrm{erg} \mathrm{cm}^{-2} \mathrm{~s}^{-1}\end{array}$ & $\chi_{v}^{2} / \mathrm{dof}$ \\
\hline \multirow[t]{5}{*}{ IGR J18457+0244 } & c*phabs*pow & $1.04_{-0.15}^{+0.17}$ & $84_{-16}^{+20}$ & $2.3 \pm 0.7$ & & & & & & $7.7 \pm 0.5$ & $1.13 / 49$ \\
\hline & c*phabs*(pow+gauss) & $1.02_{-0.14}^{+0.13}$ & $74_{-17}^{+19}$ & $1.9 \pm 0.7$ & & & $6.01_{-0.06}^{+0.04}$ & $300 \pm 150$ & $8_{-3}^{+4}$ & $4.1 \pm 0.2$ & $0.85 / 46$ \\
\hline & $c^{*}$ phabs*(cutoffpl+gauss) & $1.02_{-0.15}^{+0.16}$ & $72_{-16}^{+15}$ & $1.8_{-1.3}^{+0.9}$ & $\geq 0.01$ & & $6.01_{-0.06}^{+0.04}$ & $310 \pm 160$ & $8_{-3}^{+4}$ & $4 \pm 3$ & $0.87 / 45$ \\
\hline & c*phabs*(bbodyrad+gauss) & $1.03_{-0.15}^{+0.17}$ & $57_{-14}^{+15}$ & & & $2.1_{-0.4}^{+0.6}$ & $6.01_{-0.06}^{+0.05}$ & $310 \pm 160$ & $6_{-2}^{+3}$ & $1.4 \pm 0.2$ & $0.92 / 46$ \\
\hline & c*phabs*(comptt+gauss) & $1.02_{-0.15}^{+0.17}$ & $72_{-30}^{+19}$ & $\leq 200$ & $\leq 2.8$ & $\leq 115$ & $6.01_{-0.06}^{+0.04}$ & $300 \pm 150$ & $8_{-3}^{+4}$ & $2.2 \pm 0.3$ & $0.89 / 44$ \\
\hline \multirow[t]{5}{*}{ IGR J18462-0223 } & c*phabs*pow & $1.13 \pm 0.03$ & $28 \pm 1$ & $1.5 \pm 0.1$ & & & & & & $36.5 \pm 0.7$ & $1.32 / 337$ \\
\hline & $c^{*}$ phabs*(pow+gauss) & $1.13 \pm 0.03$ & $28 \pm 1$ & $1.5 \pm 0.1$ & & & $6.41_{-0.04}^{+0.05}$ & $70 \pm 32$ & $15 \pm 5$ & $27.7 \pm 0.6$ & $1.25 / 334$ \\
\hline & c*phabs*(cutoffpl+gauss) & $1.13 \pm 0.03$ & $21 \pm 2$ & $-0.8 \pm 0.6$ & $3.3_{-0.7}^{+1.3}$ & & $6.41 \pm 0.05$ & $48_{-27}^{+43}$ & $10.1_{-0.4}^{+0.5}$ & $16 \pm 10$ & $1.14 / 333$ \\
\hline & c*phabs*(bbodyrad+gauss) & $1.13 \pm 0.03$ & $19 \pm 1$ & & & $2.17_{-0.05}^{+0.06}$ & $6.40_{-0.05}^{+0.06}$ & $41_{-23}^{+16}$ & $8_{-4}^{+5.4}$ & $14.9 \pm 0.3$ & $1.16 / 334$ \\
\hline & c*phabs*(comptt+gauss) & $1.13 \pm 0.03$ & $21_{-3}^{+4}$ & $12_{-6}^{+5}$ & $\leq 1.7$ & $\leq 229$ & $6.41_{-0.06}^{+0.05}$ & $49_{-17}^{+44}$ & $10.3 \pm 0.5$ & $16 \pm 14$ & $1.15 / 332$ \\
\hline \multirow[t]{2}{*}{ IGR J18482+0049 } & $\mathrm{c}^{*}$ phabs* ${ }^{*}$ pow & $1.03_{-0.07}^{+0.08}$ & $44 \pm 5$ & $2.0 \pm 0.3$ & & & & & & $8 \pm 2$ & $1.01 / 108$ \\
\hline & c*phabs*bbodyrad & $1.04_{-0.07}^{+0.08}$ & $31_{-3}^{+4}$ & & & $1.9_{-0.1}^{+0.2}$ & & & & $2.4 \pm 0.4$ & $1.05 / 108$ \\
\hline \multirow[t]{5}{*}{ IGR J18532+0416 } & c*phabs*pow & $1.16 \pm 0.07$ & $3.3_{-0.3}^{+0.4}$ & $1.4 \pm 0.1$ & & & & & & $1.8 \pm 0.1$ & $1.05 / 166$ \\
\hline & c*phabs*(pow+gauss) & $1.16 \pm 0.07$ & $3.4 \pm 0.4$ & $1.4 \pm 0.1$ & & & $6.10_{-0.04}^{+0.06}$ & $253_{-109}^{+122}$ & $4_{-1}^{+2}$ & $1.8 \pm 0.1$ & $0.94 / 163$ \\
\hline & c*phabs*(cutoffpl+gauss) & $1.16 \pm 0.07$ & $3.1 \pm 0.6$ & $1.1_{-0.6}^{+0.4}$ & $\geq 6$ & & $6.10_{-0.04}^{+0.06}$ & $250 \pm 90$ & $4_{-1}^{+2}$ & $1.7 \pm 0.2$ & $0.94 / 162$ \\
\hline & c*phabs*(bbodyrad+gauss) & $1.16 \pm 0.07$ & $1.2 \pm 0.2$ & & & $1.53 \pm 0.08$ & $6.09_{-0.04}^{+0.06}$ & $207_{-77}^{+96}$ & $4_{-1}^{+2}$ & $1.2 \pm 0.1$ & $1.23 / 163$ \\
\hline & c*phabs*(comptt+gauss) & $1.17_{-0.07}^{+0.08}$ & $2.1_{-0.5}^{+1.6}$ & $1.5_{-1.2}^{+3.6}$ & $\leq 0.8$ & $\leq 294$ & $6.11_{-0.05}^{+0.06}$ & $270 \pm 110$ & $4_{-1}^{+2}$ & $\leq 6.5$ & $0.94 / 161$ \\
\hline \multirow[t]{2}{*}{ IGR J18538-0102 } & c*phabs*pow & $1.02_{-0.02}^{+0.03}$ & $1.98 \pm 0.08$ & $1.57 \pm 0.04$ & & & & & & $6.3 \pm 0.1$ & $1.33 / 359$ \\
\hline & c*phabs*cutoffpl & $1.02_{-0.02}^{+0.03}$ & $1.7 \pm 0.1$ & $1.1 \pm 0.2$ & $9_{-3}^{+5}$ & & & & & $5.6 \pm 0.8$ & $1.28 / 358$ \\
\hline
\end{tabular}

NOTE. - Errors are quoted at $90 \%$ confidence. $C$ is an instrumental cross-calibration coefficient which is fixed at 1 for pn and variable for MOS. In the Xspec formalism: phabs $=$ photoelectric absorption; pow $=$ power law; cutoffpl $=$ cutoff power law; bbodyrad $=$ radial blackbody; comptt $=$ Compton thermalization; and gauss $=$ Gaussian . 


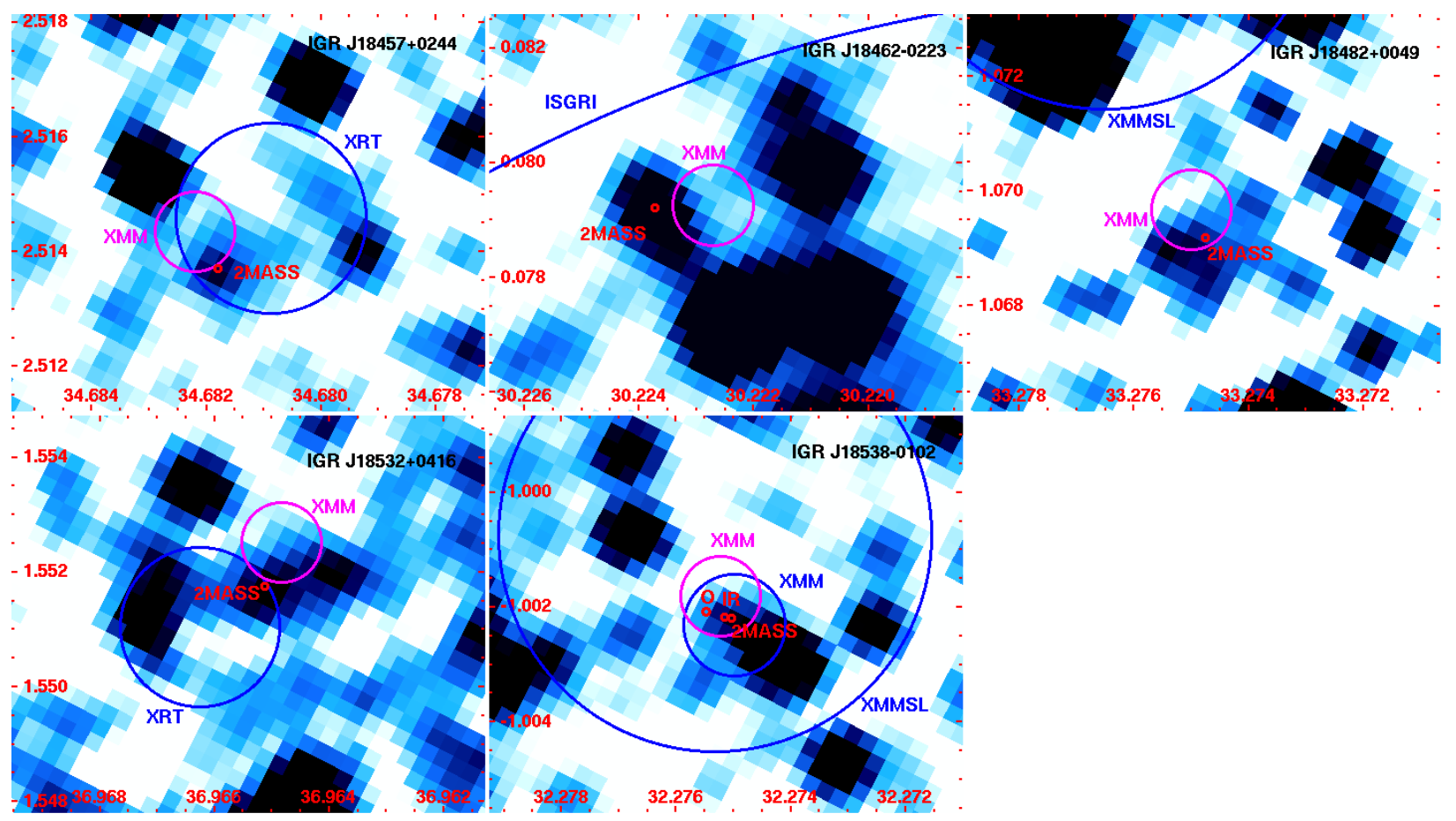

Fig. 6. - 2MASS $J$-band images of the fields of the five targets in Galactic coordinates (Skrutskie et al. 2006). The positions obtained with XMM-Newton (this work) are shown as magenta circles ( $2{ }^{\prime \prime} 5$ accuracy at $90 \%$ confidence), while the blue and red circles represent the error circles (90\% confidence) of previously proposed X-ray and optical/infrared associations, respectively. Please see the text for details.

high $N_{\mathrm{H}}$ value (which can also be found among obscured X-ray pulsars), the main evidence supporting the AGN designation is the iron line energy, and this detection is marginal since only 2 spectral bins in pn show significant deviations $(\geq 3 \sigma)$ from the continuum (1 bin for each MOS detector). On the other hand, if the potential periodic modulation can be reproduced in other observations, and if the signal were found to be coherent, it would point instead to a neutron star spin period, and the AGN would no longer be a viable explanation.

In any case, additional long-duration observations are needed to elucidate the nature of IGR J18457+0244.

\subsection{IGR J18462-0223}

Grebenev et al. (2007) discovered IGR J18462-0223 during a flare that lasted a few hours. The source was actually in outburst a year earlier but this flare had gone unnoticed until reexamination of archival data (Grebenev \& Sunyaev 2010). A day after its discovery, it could no longer be detected. The source erupted again in 2010, which led Grebenev \& Sunyaev (2010) to suggest that the source belonged to a newlyrecognized class of SGXB: a supergiant fast X-ray transient (SFXT). These are HMXBs with early-type supergiant donors feeding an X-ray source that, unlike in "classical" SGXBs, features huge variability in its emission (luminosity swings of $\sim 10^{4}-10^{5}$ are typical).

For IGR J18462-0223, we obtain with XMMNewton a position of R.A. $=18^{\mathrm{h}} 46^{\mathrm{m}} 12 \mathrm{~s} .68$ and Dec. $=-02^{\circ} 22^{\prime} 29^{\prime \prime} .3$ which is $1^{\prime} .5$ from (and still compatible with) the ISGRI position of Grebenev \& Sunyaev (2010) which has an error radius of 1'6. The 2MASS image of the field (Fig.6) shows that the nearest catalogued infrared source is located outside the $X M M$ Newton error radius (3".4 from the EPIC position): 2MASS J18461279-0222261 with magnitudes of $14.5 \pm 0.1,13.8 \pm 0.1$, and $\geq 12.7$, respectively, in the $J, H$, and $K$ bands (Skrutskie et al. 2006). This object is also listed as USNO B-1.0 0876-0579765 (Monet et al.2003), DENIS J184612.8-022226 (The DENIS Consortium 2005), and GLIMPSE G030.2231+00.0791 (Benjamin et al. 

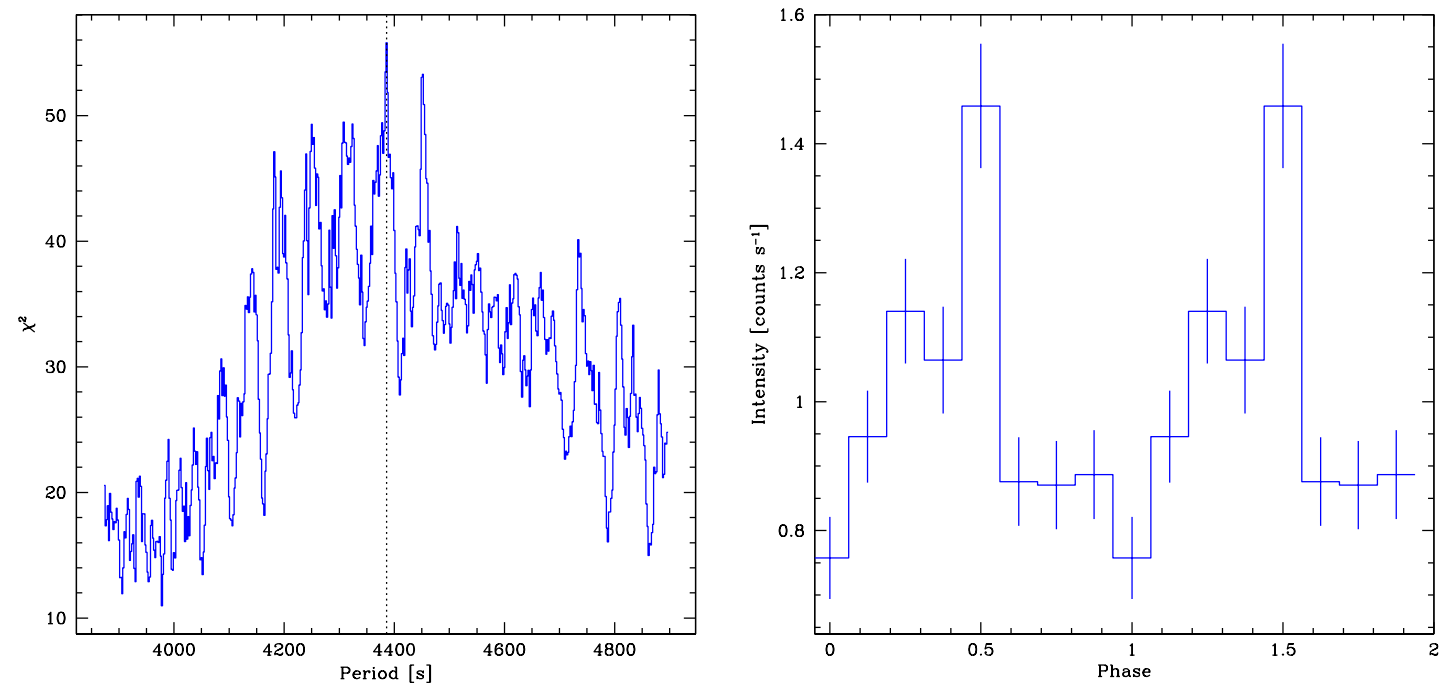

Fig. 7.- Left: Results from a periodicity search $\left(\chi^{2}\right.$ distribution) performed on the pn light curve $(0.13-15 \mathrm{keV})$ of IGR J18457+0244 centered at $4386 \mathrm{~s}$ (vertical line), with 8 bins per period, and a resolution of $2 \mathrm{~s}$. Right: Pulse profile showing two phases of IGR J18457+0244 for a period of $4386 \mathrm{~s}$. The zero phase, which corresponds to the phase where the flux is at a minimum, is MJD 55660.00609.

2003). No other objects from other wavelengths are located inside the XMM-Newton error circle.

In the X-ray spectrum of IGR J18462-0223 shown in Fig. 5, we detect a large column density $\left(N_{\mathrm{H}} \sim(2-\right.$ 3) $\times 10^{23} \mathrm{~cm}^{-2}$ ) and an iron $K \alpha$ line at $6.41_{-0.04}^{+0.05} \mathrm{keV}$ $(E W=70 \pm 32 \mathrm{eV})$. The addition of a Gaussian to the power law (to account for the $K \alpha$ line) leads to a small improvement in the quality of the fit from $\chi_{v}^{2} /$ dof $=$ $1.32 / 337$ to $1.25 / 334$. The best fit $\left(\chi_{v}^{2} / \mathrm{dof}=1.14 / 333\right)$ is obtained with the addition of a cutoff at $3.3_{-0.7}^{+1.3} \mathrm{keV}$. The resulting photon index and column density are $\Gamma=-0.8 \pm 0.6$ and $N_{\mathrm{H}}=(21 \pm 2) \times 10^{22} \mathrm{~cm}^{-2}$, respectively. A blackbody (bbodyrad) also provides a good fit to the $\mathrm{X}$-ray spectra $\left(\chi_{v}^{2} / \mathrm{dof}=1.16 / 334\right)$. For a Compton thermalization model (compTT), the plasma temperature and energy of the seed photons could not be constrained.

The spectral shape is reminiscent of other windaccreting X-ray binaries discovered by INTEGRAL, particularly that of the obscured SGXB pulsar in the Norma Arm IGR J16393-4643 (Bodaghee et al. 2006): both sources have large $N_{\mathrm{H}}$ values, hard power law continua, iron line(s), and low-energy cutoffs. It is important to note that the cutoff energy in IGR J18462-0223 is below $20 \mathrm{keV}$. This cutoff is required since the photon index in the ISGRI band (20-
$60 \mathrm{keV}$ ) is $\Gamma=2.5 \pm 0.3$ (Grebenev \& Sunyaev 2010), i.e., steeper than the slope of $1.5 \pm 0.1$ that we measure for an absorbed power law between 0.5 and $10 \mathrm{keV}$. In HMXB systems, such low cutoff energies are typical of accreting neutron stars (Nagase 1989).

Therefore, we searched for periodic modulations in the pn light curve of IGR J18462-0223 and we found a coherent pulsation at a period of $997 \pm 1 \mathrm{~s}$ with $\chi^{2}=162$ for 10 bins per trial period $(\sim 12 \sigma$ significance, not corrected for the number of trials). The pulse profile folded on a period of $997 \mathrm{~s}$ and beginning at MJD 55669.00808(1) is shown in Fig.8 The pulse fraction is $12 \% \pm 2 \%$. Thus, the compact object hosted by IGR J18462-0223 is an accreting neutron star whose magnetic and spin axes are misaligned.

The SFXT designation for IGR J18462-0223 rests on its sporadic detection history in the hard X-rays Grebenev \& Sunyaev 2010). In our 30-ks long and continuous observation (Fig.44, we find no evidence of the large variability associated with this class of objects. The dynamic range for the $0.13-15-\mathrm{keV}$ flux is an order of magnitude or less. This is more typical of persistently-emitting SGXB systems. Assuming that the source resides within the Scutum Arm whose tangent is $\sim 7 \mathrm{kpc}$ away (e.g., Russeil 2003), its unabsorbed flux $(0.5-10 \mathrm{keV})$ of $1.6 \times 10^{-11} \mathrm{erg} \mathrm{cm}^{-2} \mathrm{~s}^{-1}$ 

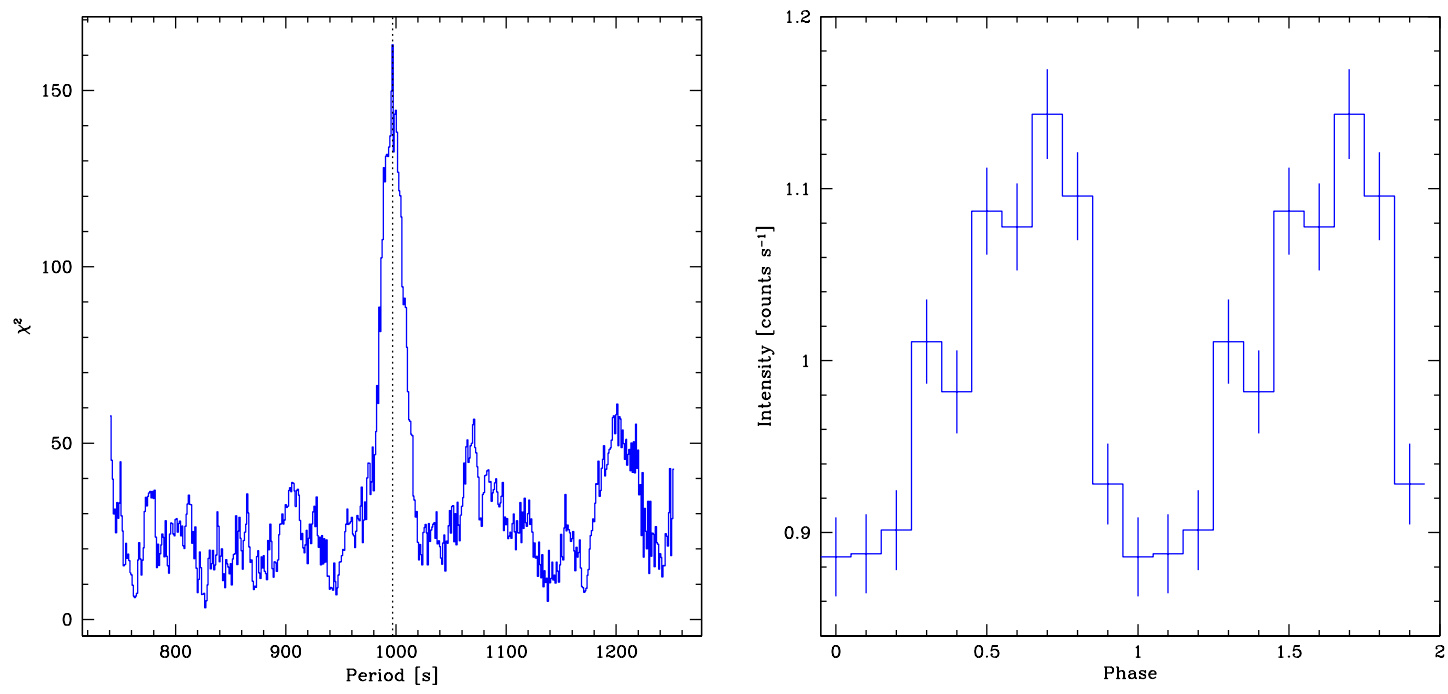

Fig. 8. - Left: Results from a periodicity search $\left(\chi^{2}\right.$ distribution) performed on the pn light curve $(0.13-15 \mathrm{keV})$ of IGR J18462-0223 centered at $997 \mathrm{~s}$ (vertical line), with 10 bins per period, and a resolution of $1 \mathrm{~s}$. Right: Pulse profile showing two phases of IGR J18462-0223 for a period of $997 \mathrm{~s}$. The zero phase (phase in which the flux is at a minimum) corresponds to MJD 55669.00808.

converts to a luminosity of around $10^{35} \mathrm{erg} \mathrm{s}^{-1}$. This is a typical luminosity for an SFXT in the active state (e.g., Romano et al. 2011), but it is an order of a magnitude lower than in other persistently-emitting SGXBs (e.g., Walter et al. 2006), unless the source were located more than $7 \mathrm{kpc}$ from us.

Overall, the timing and spectral characteristics of IGR J18462-0223 suggest that this system is an obscured (and probably distant) SGXB pulsar, and one of the few examples of a highly-obscured HMXB in the direction of the Scutum Arm. The source could represent an intermediate SGXB-SFXT system like IGR J16479-4514, which would make IGR J18462-0223 the seventh (out of 20 known SFXT candidates) to have a measured spin period.

\subsection{IGR J18482+0049}

Our XMM-Newton observation provides us with the most accurate X-ray position for IGR J18482+0049: R.A. $=18^{\mathrm{h}} 48^{\mathrm{m}} 15.32$, and Dec. $=+00^{\circ} 47^{\prime} 34^{\prime \prime} \cdot 9$. This position is compatible with the discovery position of ISGRI (Bird et al. 2010) which is 2'.5 away and has an error radius of 4:4. Stephen et al. (2010) proposed an association of IGR J18482+0049 with an object from the XMM-Newton Slew Survey (XMMSL:
Saxton et al. 2008) that is located $2 ! 2$ from the ISGRI position and has a 90\%-confidence radius of 13".6. However, our XMM-Newton position is $21^{\prime \prime}$ from the XMMSL source which is henceforth ruled out. Only one object in the 2MASS catalog is consistent with the XMM-Newton position: 2MASS J18481540+0047332, which is located $2, .0$ from the center of the $X M M$ Newton error circle and has magnitudes of $\geq 15.8$, $\geq 14.0$, and $13.9 \pm 0.1$, respectively, in the $J, H$, and $K$ bands (Skrutskie et al. 2006). This source is also listed as GLIMPSE G033.2747+01.0692 Benjamin et al. 2003). There are no other objects from other wavelengths inside the XMM-Newton error circle.

An absorbed power law is sufficient to model the X-ray spectrum of IGR J18482+0049 (Fig[5). The column density is large $\left(N_{\mathrm{H}} \sim 4 \times 10^{23} \mathrm{~cm}^{-2}\right)$ and is well in excess of the cumulative line-of-sight absorption value (Kalberla et al. 2005), and so favors an interpretation in which most of the obscuring material is intrinsic to the system. The photon index is $\Gamma=2.0 \pm 0.3$ with an unabsorbed flux of $7.6 \times 10^{-12} \mathrm{erg} \mathrm{cm}^{-2} \mathrm{~s}^{-1}$ in the $0.5-10-\mathrm{keV}$ band $\left(=9.1 \times 10^{34}\left[\frac{d}{10 \mathrm{kpc}}\right]^{2} \mathrm{erg} \mathrm{s}^{-1}\right)$. This simple model provides an excellent fit $\left(\chi_{v}^{2} / \mathrm{dof}\right.$ $=1.01 / 108)$. An absorbed blackbody with a temperature of $1.9_{-0.1}^{+0.2} \mathrm{keV}$ also provides a good fit $\left(\chi_{v}^{2} / \mathrm{dof}\right.$ 

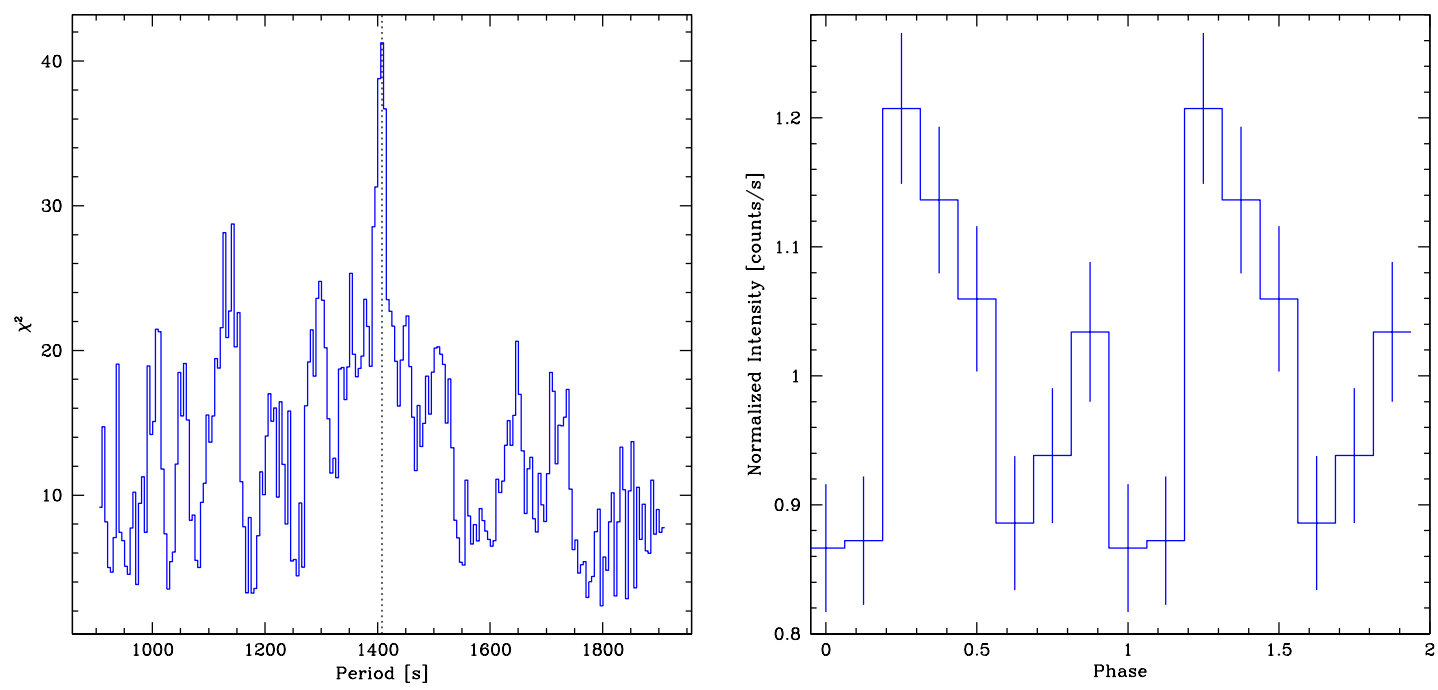

Fig. 9.- Left: Periodicity search $\left(\chi^{2}\right.$ distribution) on the pn light curve $(0.13-15 \mathrm{keV})$ of IGR J18532+0416 centered at $1408 \mathrm{~s}$ (vertical line), with 8 bins per period, and a resolution of $5 \mathrm{~s}$. Right: Pulse profile showing two phases of IGR J18532+0416 for a period of $1408 \mathrm{~s}$. The zero phase (phase with the minimum flux) corresponds to MJD 55663.00815.

$=1.05 / 108)$, but residuals appear above $10 \mathrm{keV}$. More complicated spectral models (i.e., cutoff power laws, and Comptonization) lead to unconstrained spectral parameters. We tested for the presence of a soft excess by adding a blackbody component to the absorbed power law. The addition of this component with a free temperature did not improve the fit, but holding the temperature at $0.1 \mathrm{keV}$ led to a marginal improvement over the absorbed power law $\left(\chi_{v}^{2} / \mathrm{dof}=0.99 / 107\right.$; F-test probability of $5 \%$ ) with the other spectral parameters $\left(N_{\mathrm{H}}\right.$ and $\left.\Gamma\right)$ remaining consistent.

Given the shape of the spectral continuum and the large absorbing column, its location close to the Galactic Plane, and its persistent emission in the hard Xrays, we conclude that IGR J18482+0049 is most likely a new obscured HMXB in the Scutum Arm. Many sources in this class feature pulsation periods in the X-rays, but we did not find conclusive evidence for a periodic signal between $1 \mathrm{~s}$ and $5000 \mathrm{~s}$ in the the light curve of IGR J18482+0049. Confirmation of the HMXB nature of IGR J18482+0049 must await spectral analysis of the candidate optical/IR counterpart proposed here.

\subsection{IGR J18532+0416}

Bird et al. (2010) discovered IGR J18532+0416, and the source was followed up by Swift-XRT which provided a refined X-ray position (Fiocchi et al. 2011). The position that we obtain from our XMM-Newton observation is R.A. $=18^{\mathrm{h}} 53^{\mathrm{m}} 15 \mathrm{~s} 83$, and Dec. $=$ $+04^{\circ} 17^{\prime} 48^{\prime \prime}$.5. Figure 6 shows that this position is $77^{\prime \prime 3}$ away from (but still statistically compatible with) the location of XRT Source \#2 in Fiocchi et al. (2011). The nearest potential counterpart across all wavelengths is 2MASS J18531602+0417481 which is located 2 !' 9 away. It has magnitudes of $\geq 16.5,15.2 \pm 0.1$, and $13.9 \pm 0.1$ in the $J, H$, and $K$ bands, respectively (Skrutskie et al. 2006).

A power law $(\Gamma=1.4 \pm 0.1)$ with moderate absorption $\left(N_{\mathrm{H}}=(1.98 \pm 0.08) \times 10^{22} \mathrm{~cm}^{-2}\right)$ provides a good fit to the source spectrum $\left(\chi_{v}^{2} / \mathrm{dof}=1.05 / 166\right)$. Residuals can be seen near $6 \mathrm{keV}$ (Fig. 5) so we included a Gaussian at $6.10_{-0.04}^{+0.06} \mathrm{keV}\left(E W=253_{-109}^{+122} \mathrm{eV}\right)$ which yields a fit with $\chi_{v}^{2} / \mathrm{dof}=0.94 / 163$. This suggests a redshifted iron $K \alpha$ line source at $z=0.05(1)$. The unabsorbed flux $(0.5-10 \mathrm{keV})$ is $1.8 \times 10^{-12} \mathrm{erg} \mathrm{cm}^{-2} \mathrm{~s}^{-1}$ which converts to a luminosity of $2.1 \times 10^{34}\left[\frac{d}{10 \mathrm{kpc}}\right]^{2} \mathrm{erg} \mathrm{s}^{-1}$. The implied (unabsorbed) luminosity at $z=0.05(1)$ would be $\sim 10^{43} \mathrm{erg} \mathrm{s}^{-1}\left(H_{\circ}=70 \mathrm{~km} \mathrm{~s}^{-1} \mathrm{Mpc}^{-1}\right)$. Us- 
ing a cutoff power law or a Compton thermalization model gave fits with acceptable $\chi_{v}^{2}$ values but left important parameters unconstrained (e.g., cutoff energy and plasma temperature). A blackbody model shows residuals at low and high energies and provides a poor fit overall.

The light curve for IGR J18532+0416 is presented in Fig.4 The source is soft with a hardness ratio that is negative during most of the observation. The $\chi^{2}$ distribution for the pn light curve (Fig. 9) contains a weak potential period at $1408 \mathrm{~s}\left(\chi^{2}=41\right.$ for 8 bins per trial, i.e., 5- $\sigma$ significance, not corrected for the number of trials). This candidate signal is not present in the Lomb-Scargle periodogram nor in the power spectrum. If this periodic signal is real and coherent, it would suggest an accreting neutron star in an HMXB. On the other hand, if the iron line is real, then it would invalidate the pulsar hypothesis (ascribing the candidate signal to chance or to aperiodic variability on a 1-ks timescale) and would suggest either a redshifted line from material in a lowinclination disk around a black hole in an X-ray binary (e.g., Fabian et al. 1989; van der Woerd et al. 1989), or a source located at a cosmological distance (i.e., an AGN). Just as with IGR J18457+0244, if both the iron line and modulation are real, then the latter could be the signature of a low-frequency QPO from an AGN. In other words, we can not firmly establish the nature of IGR J18532+0416 because of the low signalto-noise ratio of both the iron line and the periodicity. Confirmation (or refutation) of either of these observables will require additional observations.

\subsection{IGR J18538-0102}

IGR J18538-0102 was listed as a new source in Bird et al. (2010). Stephen et al. (2010) noted the positional association with G 32.1-0.9 (Folgheraiter et al. 1997), a candidate supernova remnant (SNR) located 0 '6 from the ISGRI position. Given that the spectrum of the IGR source is harder and more absorbed than that of the SNR, Stephen et al. (2010) concluded that IGR J18538-0102 is probably a distant Galactic source or an extragalactic source, with only a coincidental association with G 32.1-0.9. Subsequently, an archival XMM-Newton observation of the field which contained the source was reanalyzed by Halpern \& Gotthelf (2010) who found a persistent source at the following coordinates: R.A. $=18^{\mathrm{h}} 53^{\mathrm{m}} 48.50$, and Dec. $=-01^{\circ} 02^{\prime} 30^{\prime \prime} \cdot 0$. This source is also listed as 2XMM J185348.4-010229
Watson et al. 2009). Inside its error circle of 3 !'2, there is a single catalogued infrared source, 2MASS J18534847-0102295, which has magnitudes of $\geq 14.2,14.0 \pm 0.1$, and $12.5 \pm 0.1$, respectively, in the $J, H$, and $K$ bands Skrutskie et al. 2006). Among multiple optical counterpart candidates present within the XMM-Newton error circle, Lutovinov et al. (2012) identified a faint source whose spectrum shows a broad $\mathrm{H} \alpha$ line corresponding to a Sey-1 AGN at $z=0.145(1)$.

Our X-ray position for IGR J18538-0102 is R.A. $=18^{\mathrm{h}} 53^{\mathrm{m}} 48.42$, and Dec. $=-01^{\circ} 02^{\prime} 28^{\prime \prime} \cdot 3$. This is 2 ".1 and 1".5 away from, but still compatible with, the XMM-Newton positions of Halpern \& Gotthelf (2010) and Malizia et al. (2010), respectively. Compared to these previously-reported XMM-Newton positions, our position is closer ( 1 '!2 away) to the optical counterpart (Sey-1) identified by Lutovinov et al. (2012), and it remains compatible with the 2MASS source which is located 1".6 away, the optical/IR counterpart (1".4 offset) listed in Halpern \& Gotthelf (2010), and with USNOB1.0 0889-0406090 (Monet et al. 2003). With a 13".6 uncertainty radius (Saxton et al. 2008), the position of the XMMSL source listed in Stephen et al. (2010) encompasses our XMM-Newton error circle (Fig.66).

The X-ray spectrum of the source (Fig.5) can be adequately described $\left(\chi_{v}^{2} / \mathrm{dof}=1.33 / 359\right)$ by a power law with moderate absorption: $N_{\mathrm{H}}=(1.98 \pm 0.08) \times$ $10^{22} \mathrm{~cm}^{-2}$. This is slightly larger than the $N_{\mathrm{H}}$ measured by Halpern \& Gotthelf (2010) and by Malizia et al. (2010), but the photon index $(\Gamma=1.57 \pm 0.04)$ and unabsorbed $0.5-10-\mathrm{keV}$ flux $\left(6.3 \times 10^{-12} \mathrm{erg} \mathrm{cm}^{-2} \mathrm{~s}^{-1}\right)$ are in agreement. Adding a cutoff at $9_{-3}^{+5} \mathrm{keV}$ leads to a small improvement in the fit $\left(\chi_{v}^{2} / \mathrm{dof}=1.28 / 358\right)$. Replacing the power law with a radial blackbody or a thermal Comptonization model leads to large residuals and poor fits.

No periodic signal could be found in the 1-5000s range in the pn light curve of IGR J18538-0102. The photon index that we measured is consistent with the AGN classification proposed by Lutovinov et al. (2012), and the column density is larger than the cumulative line-of-sight value indicating a distant or extragalactic source.

\section{Summary \& Conclusions}

In this work, we have analyzed XMM-Newton observations of five unclassified hard X-ray sources located towards the Scutum Arm. Refined X-ray positions, soft X-ray light curves, and spectral energy 
Table 4: Proposed classifications for the five sources in this study.

\begin{tabular}{lc}
\hline \hline Source Name & Classification \\
\hline IGR J18457+0244 & probable AGN, HMXB possible \\
IGR J18462-0223 & absorbed SFXT pulsar \\
IGR J18482+0049 & absorbed HMXB \\
IGR J18532+0416 & AGN? HMXB? \\
IGR J18538-0102 & probable Sey-1 \\
\hline
\end{tabular}

distributions have been derived for all five targets (Tables 23 .

Our results indicate that IGR J18462-0223 and IGR J18482+0049 are probably new heavily-absorbed $\left(N_{\mathrm{H}}>10^{23} \mathrm{~cm}^{-2}\right)$ high-mass X-ray binaries (HMXBs). The former is a slow X-ray pulsar $(P=997 \pm 1 \mathrm{~s})$ in a supergiant fast X-ray transient (SFXT) system. This makes IGR J18462-0223 the seventh SFXT (in a class composed of $\sim 20$ members) to have confirmed X-ray pulsations, providing a useful laboratory to test SFXT emission mechanisms which assume highly-magnetized ( $B \gtrsim 10^{13} \mathrm{G}$ ) neutron stars (e.g., Grebenev \& Sunyaev 2007; Bozzo et al. 2008). These two additional systems represent a $40 \%$ increase in the number of known obscured HMXBs in this region (Fig.11), helping to reduce (somewhat) the asymmetry that we continue to observe between the large number of obscured HMXB systems found towards the Norma Arm compared with the low number found in the direction of the Scutum Arm (Bodaghee et al. 2007).

We can not conclusively determine the nature of the three other targets in our sample, IGR J18457+0244, IGR J18532+0416, and IGR J18538-0102. We propose that IGR J18457+0244 and IGR J18532+0416 are probably active galactic nuclei (AGN) viewed through the plane of the Milky Way, but Galactic X-ray binaries can not be ruled out. For IGR J18538-0102, the spectral parameters (photon index and column density) are consistent with an AGN interpretation.

Confirmation of the classifications for all sources in this study (see Table 4) will require further observations of the optical/infrared counterparts which we propose here.

The authors thank the anonymous referee whose constructive review led to significant improvements in the manuscript. This research has made use of: observations obtained with XMM-Newton, an ESA science mission with instruments and contributions directly funded by ESA Member States and NASA; data obtained from the High Energy Astrophysics Science Archive Research Center (HEASARC) provided by NASA's Goddard Space Flight Center; the SIMBAD database operated at CDS, Strasbourg, France; NASA's Astrophysics Data System Bibliographic Services; the Two Micron All Sky Survey, which is a joint project of the University of Massachusetts and the Infrared Processing and Analysis Center/California Institute of Technology, funded by the National Aeronautics and Space Administration and the National Science Foundation; and the IGR Sources page maintained by JR and AB (http://irfu.cea.fr/Sap/IGR-Sources).

Facilities: XMM-Newton.

\section{REFERENCES}

Balucinska-Church, M., \& McCammon, D. 1992, ApJ, 400, 699

Benjamin, R. A., et al. 2003, PASP, 115, 953

Bird, A. J., et al. 2010, ApJS, 186, 1

Bodaghee, A., Walter, R., Zurita Heras, J. A., Bird, A. J., Courvoisier, T. J.-L., Malizia, A., Terrier, R., \& Ubertini, P. 2006, A\&A, 447, 1027

Bodaghee, A., et al. 2007, A\&A, 467, 585

Bozzo, E., Falanga, M., \& Stella, L. 2008, ApJ, 683, 1031

Dickey, J. M., \& Lockman, F. J. 1990, ARA\&A, 28, 215

Espaillat, C., Bregman, J., Hughes, P., \& LloydDavies, E. 2008, ApJ, 679, 182

Fabian, A. C., Rees, M. J., Stella, L., \& White, N. E. 1989, MNRAS, 238, 729

Fiocchi, M., Landi, R., Bassani, L., Bazzano, A., Bird, A. J., Gehrels, \& Kennea, J. A. 2011, The Astronomer's Telegram, 3256, 1

Folgheraiter, E. L., Warwick, R. S., Watson, M. G., \& Koyama, K. 1997, MNRAS, 292, 365

Gierliński, M., Middleton, M., Ward, M., \& Done, C. 2008, Nature, 455, 369

Grebenev, S. A., Revnivtsev, M. G., \& Sunyaev, R. A. 2007, The Astronomer's Telegram, 1319, 1 
Grebenev, S. A., \& Sunyaev, R. A. 2007, Astronomy Letters, 33, 149

—. 2010, Astronomy Letters, 36, 533

Gupta, A. C., Srivastava, A. K., \& Wiita, P. J. 2009, ApJ, 690, 216

Halpern, J. P., \& Gotthelf, E. V. 2010, The Astronomer's Telegram, 2445, 1

Horne, J. H., \& Baliunas, S. L. 1986, ApJ, 302, 757

Jansen, F., et al. 2001, A\&A, 365, L1

Kalberla, P. M. W., Burton, W. B., Hartmann, D., Arnal, E. M., Bajaja, E., Morras, R., \& Pöppel, W. G. L. 2005, A\&A, 440, 775

Krivonos, R., Tsygankov, S., Revnivtsev, M., Grebenev, S., Churazov, E., \& Sunyaev, R. 2010, A\&A, 523, A61

Lachowicz, P., Gupta, A. C., Gaur, H., \& Wiita, P. J. 2009, A\&A, 506, L17

Landi, R., Malizia, A., Bazzano, A., Fiocchi, M., Bird, A. J., Gehrels, N., \& Burrows, D. N. 2010, The Astronomer's Telegram, 3078, 1

Leahy, D. A., Darbro, W., Elsner, R. F., Weisskopf, M. C., Kahn, S., Sutherland, P. G., \& Grindlay, J. E. 1983, ApJ, 266, 160

Liu, Q. Z., van Paradijs, J., \& van den Heuvel, E. P. J. 2000, A\&AS, 147, 25

Lutovinov, A. A., Burenin, R. A., Revnivtsev, M. G., \& Bikmaev, I. F. 2012, Astronomy Letters, 38, 1

Malizia, A., Bassani, L., Sguera, V., Stephen, J. B., Bazzano, A., Fiocchi, M., \& Bird, A. J. 2010, MNRAS, 408, 975

Matt, G., \& Guainazzi, M. 2003, MNRAS, 341, L13

Monet, D. G., et al. 2003, AJ, 125, 984

Nagase, F. 1989, PASJ, 41, 1

Press, W. H., \& Rybicki, G. B. 1989, ApJ, 338, 277

Rodriguez, J., Tomsick, J. A., \& Bodaghee, A. 2010, A\&A, 517, A14

Romano, P., et al. 2011, MNRAS, 410, 1825

Russeil, D. 2003, A\&A, 397, 133
Saxton, R. D., Read, A. M., Esquej, P., Freyberg, M. J., Altieri, B., \& Bermejo, D. 2008, A\&A, 480, 611

Skrutskie, M. F., et al. 2006, AJ, 131, 1163

Stephen, J. B., Landi, R., Masetti, N., Fiocchi, M., Capitanio, F., Bird, A. J., \& Clark, D. J. 2010, The Astronomer's Telegram, 2441, 1

Strüder, L., et al. 2001, A\&A, 365, L18

The DENIS Consortium. 2005, VizieR Online Data Catalog, 2263, 0

Turner, M. J. L., et al. 2001, A\&A, 365, L27

Vallée, J. P. 2008, ApJ, 681, 303

van der Klis, M. 1989, in Timing Neutron Stars, ed. H. Ögelman \& E. P. J. van den Heuvel, 27

van der Woerd, H., White, N. E., \& Kahn, S. M. 1989, ApJ, 344, 320

Walter, R., et al. 2006, A\&A, 453, 133

Watson, M. G., et al. 2009, A\&A, 493, 339

Wilms, J., Allen, A., \& McCray, R. 2000, ApJ, 542, 914

This 2-column preprint was prepared with the AAS LATEX macros v5.2. 